\title{
Catalysis for new energy resources and environmental protection
}

This issue of Catalysis Today is a selection of the papers, concerning catalysis for new energy resources and environmental protection, presented at the 3rd Conference of the Indo-Pacific Catalysis Association and The 21st Taiwan Symposium on Catalysis and Reaction Engineering (IPCAT-3 and TSCRE-21) at National Taiwan University, Taipei, Taiwan on November 16-18, 2003. IPCAT-3 aims to provide an opportunity to close-link and stimulate the cooperation among catalysis researchers from different countries, especially in the Indo-Pacific Rim. Annual TSCRE is the major event for scientists and engineers in Taiwan, whose research focus on the areas of catalysis and reaction engineering. The joint conferences were held under the auspices of Academia Sinica of Taiwan, National Taiwan University, Indo-Pacific Catalysis Association and Taiwan Catalysis Society, and had about 240 participants from 18 countries.

As in most of the developed areas in the world, some countries in the Indo-Pacific areas are suffering from the effects of energy shortage and the effects of pollution accompanying industrialization. The environmental protection and the alternative energy resources are becoming big issues in every society. Therefore, the 15 papers of this volume focus on new energy resources and environmental protection. It tries to emphasize that the new developments of sustainable energy and environmental benign technology have become one of crucial research area in catalysis. Global warming is a serious issue due to human activity by consuming fossil fuel. One of sustainable-energy developments is to utilize unlimited solar energy via photo catalysis, including water splitting and $\mathrm{CO}_{2}$ transformation. Moreover, the fuel cell provides a highly efficient way to convert chemical to electrical energy so that energy consumption can be significantly decreased. The catalytic reforming plays an important role in hydrogen supply for fuel cell. Also, the catalytic process has been employed to reduce air pollution for decades. The recent progress on the remedy of volatile organic compounds (VOC), $\mathrm{NO}_{x}$ and halogenated compounds are covered in this volume. It is hoped that the materials presented in this volume will be valuable for the scientific and technical community and will stimulate future researches in new energy and environmental protection.

Finally, we thank the participators of the IPCAT- 3 and TSCRE-21 and the reviewers who carried out the refereeing process. We also express our gratitude to the journal Editor (Professor J.J. Spivey) and the colleagues in Log-in Department of Elsevier (Dr. Laura Hassink and Ms. Sandra Korver) for their supports and assistance to this special issue. The financial supports of Academia Sinica, Ministry of Education, National Science Council, Chinese Chemical Society, Institute of Chinese Chemical Engineers, Industrial Technology Research Institute, Chinese Petroleum Corporation and the CTCI Foundation in Taiwan, are highly appreciated.

Jeffrey C.S. $\mathrm{Wu}^{*}$ Ben-Zu Wan ${ }^{1}$

Department of Chemical Engineering National Taiwan University, Taipei 10617, Taiwan ROC *Corresponding author. Tel.: +886 223631994 fax: +886223623040

E-mail addresses: cswu@ntu.edu.tw (J.C.S. Wu) benzuwan@ntu.edu.tw (B.-Z. Wan)

${ }^{1}$ Tel.: +886 2 33663021; fax: +886223623040

Available online 31 July 2004 